\title{
Familial cerebral cavernous malformation
}

INSERM

\section{Source}

INSERM. (1999). Orphanet: an online rare disease and orphan drug data base. Familial cerebral cavernous malformation. ORPHA:221061

Familial cerebral cavernous malformation (FCCM) is a rare evolutive vascular malformation disorder characterized by closely clustered irregular dilated capillaries that can be asymptomatic or that can cause variable neurological manifestations such as seizures, non-specific headaches, progressive or transient focal neurologic deficits, and/or cerebral hemorrhages. 